\title{
First Experiments in the Robotic Investigation of Life in the Atacama Desert of Chile
}

\author{
David Wettergreen, Nathalie Cabrol ${ }^{1}$, James Teza, Paul Tompkins, \\ Chris Urmson, Vandi Verma ${ }^{1}$, Michael Wagner, William Whittaker \\ The Robotics Institute \\ Carnegie Mellon University \\ Pittsburgh, PA 15213-3891 USA
}

\begin{abstract}
The Atacama Desert of northern Chile may be the most lifeless place on Earth, yet where the desert meets the Pacific coastal range desiccation-tolerant micro-organisms are known to exist. The gradient of biodiversity and habitats in the Atacama's subregions remain unexplored and are the focus of the Life in the Atacama project. To conduct this investigation, long traverses must be made across the desert with instruments for geologic and biologic measurements.

In this paper we motivate the Life in the Atacama project from both astrobiologic and robotic perspectives. We focus on some of the research challenges we are facing to enable endurance navigation, resource cognizance, and long-term survivability. We conducted our first scientific investigation and technical experiments in Chile with the mobile robot Hyperion. We describe the experiments and the results of our analysis. These results give us insight into the design of an effective robotic astrobiologist and into the methods by which we will conduct scientific investigation in the next field season.
\end{abstract}

\section{INTRODUCTION}

Astrobiology is the study of the living universe and it addresses the realization that the origin and evolution of life itself cannot be understood unless it is examined in all the environments in which life might exist. Astrobiologic investigations search for planets of other stars, evidence of life on Mars, as well as life at extremes here on Earth.

The Atacama Desert is the most arid region on Earth and in several ways analogous to Mars. Evidence suggests that the interior of the Atacama is lifeless, yet where the desert meets the Pacific coastal range dessication-tolerant microorganisms are known to exist. The gradient of biodiversity and habitats in the Atacama's subregions remain unexplored and are the focus of the Life in the Atacama project.

Our field investigation is bringing new scientific understanding of the Atacama as a habitat for life through the creation of robotic astrobiology. This involves capabilities for autonomously traversing tens to hundreds of kilometers while deploying sensors to survey the varying geologic and biologic properties of the environment, Fig. 1. Our goal is to make genuine discoveries about the limits of

${ }^{1}$ Currently with NASA Ames Research Center, Mountain View, CA, USA

The Life in the Atacama project is supported at Carnegie Mellon by the NASA Astrobiology Science and Technology for Exploring the Planets (ASTEP) program under contract NAG5-12890.

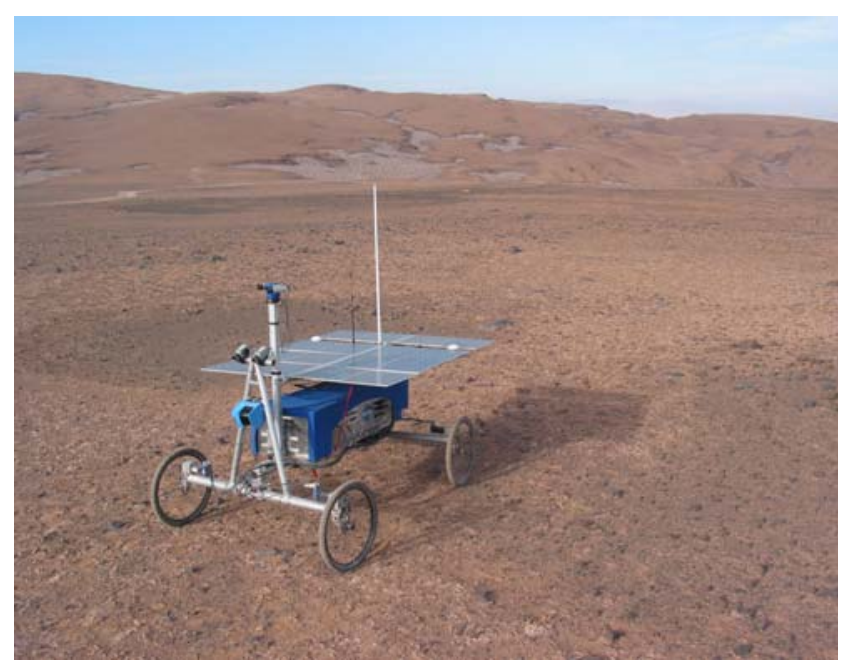

Figure 1. Hyperion rover in the Atacama Desert, Chile

life on Earth and to generate knowledge about life in extreme environments that can be applied to future planetary missions. Through these experiments we also hope to develop and practice the methods by which a rover might best be employed to survey desert terrain in search of the habitats in which life can survive.

\section{AtACAMA DeSERT}

The Atacama Desert in northern Chile lies between the Pacific coastal range and the Andes. It is the driest desert on Earth, drier even than the Antarctic plateau, for in some regions there has been no measurable precipitation in centuries. In regions along the western coast fog, called camanchacas, occasionally penetrates through the coastal mountains to reach the desert and in the east snowmelt from the Andes feeds an aquifer deep below the desert.

The Atacama Desert presents an excellent analog to Mars because it is extremely dry but

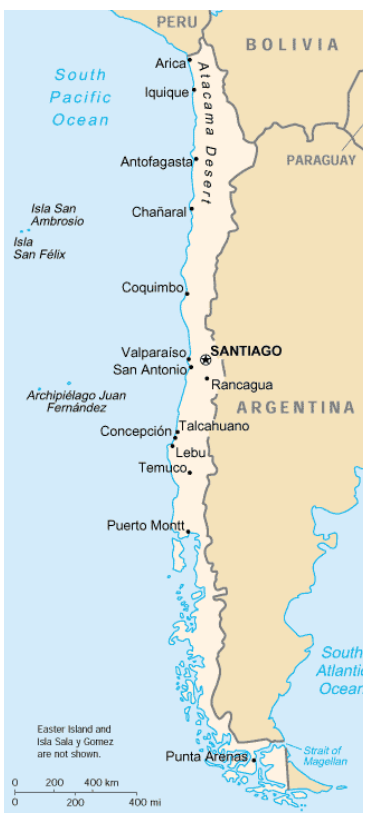


also, like Mars, the desert experiences very high UV radiation. This is because it is at high altitude $700-4000 \mathrm{~m}$ above sea level and the atmosphere is particularly transparent due to the minimal of water vapor. Lastly, the soils in the Atacama have been found to be particularly high in oxidants leading to the rapid breakdown of organic material. [1] A similar discovery was made by the Viking landers on Mars in their soil analysis. [2] The result is that in some regions of desert almost no biogenic material can be found, including no culturable spores, despite the continuous infall of biogenic material occurring globally from the atmosphere.

For these reasons: aridity, high UV radiation and soil oxidants the Atacama is analogous to Mars. Of course, the Atacama is not uniform or constant in these properties. There is variation in aridity between the central desert and its boundaries along the coast and Andes, UV radiation varies with season and altitude, and the composition of the soil is locally determined by mineralogical content.

The study of life in the Atacama tells us more about living organisms and their adaptation to this extreme environment here on Earth as well as potential habitats or at least areas for investigation on Mars.

\section{SCIENTIFIC AND TECHNOLOGICAL OBJECTIVES}

Our field investigations will use a rover to make controlled transects in the Atacama with instruments to detect life and to characterize habitats. The first year's field investigation and experiments, described here, validated components and methods, the second year will test the functional integration of the system with the necessary capabilities for exploration, and in the third year we will conduct a fully operational science mission.

For the Life in the Atacama project we have set objectives in areas of science and technology.

\section{A. Science}

Site-specific studies have found microorganisms at varying concentrations in some areas of the Atacama.The distribution of these organisms and the boundary conditions for each habitat, if they exist, remain unknown. The specific scientific objective of this work is to map the distribution of life in the Atacama, and to accomplish this by robotic traverse, Fig. 2. Our goals are to:

Seek Life: Detect life unambiguously through confirming lines of evidence; characterize biota surviving in the habitat; measure spatial variability of biodiversity.

Understand Habitat: Determine the physical and environmental conditions associated with identified habitats and identify boundary conditions of microorganic life; examine biological oases and microbe communities and measure diversity of habitats for microorganic life.

Make Relevant Measurements: Integrate and field-test instruments that form an appropriate science payload; make measurements that motivate the exploration of analogous environments on Mars; develop methods and procedures for conducting long-distance science surveys.

\section{B. Technology}

To achieve our science objectives we must deploy lifedetection instruments with an rover capable of long-distance traverse. We must create a robotic astrobiologist that can:

Navigate Over the Horizon: Navigate beyond the robot field-of-view $(>1 \mathrm{~km})$; model the environment and detect obstacles at necessary scales; localize based on odometry, sun position, and local feature/global landmark tracking (but not artificial satellites); register observations to orbital datasets and limit position error to $5 \%$ of distance traveled

Use Resources Efficiently: Enable onboard, resourcelimited traverse planning and sequence execution to address: Power, solar and battery energy and overnight hibernation; Communication, cycles, delay, and data volume and Science: instrument use and sampling requests

Enable Autonomy with Self-Awareness: Establish variable rover autonomy and effective remote investigation (telescience) over low-bandwidth, long-latency communication links; develop rover self-awareness, monitoring hardware and software for fault detection and recovery; achieve multi-day unattended operation.

\section{HYPERION}

For our first field season, testing component of robotics technologies for the Atacama investigation, we modified Hyperion, a solar-powered rover that was designed to exploit the advantages of sun-synchrony.[3] Hyperion represents a class of polar robot notable for reduced mass,

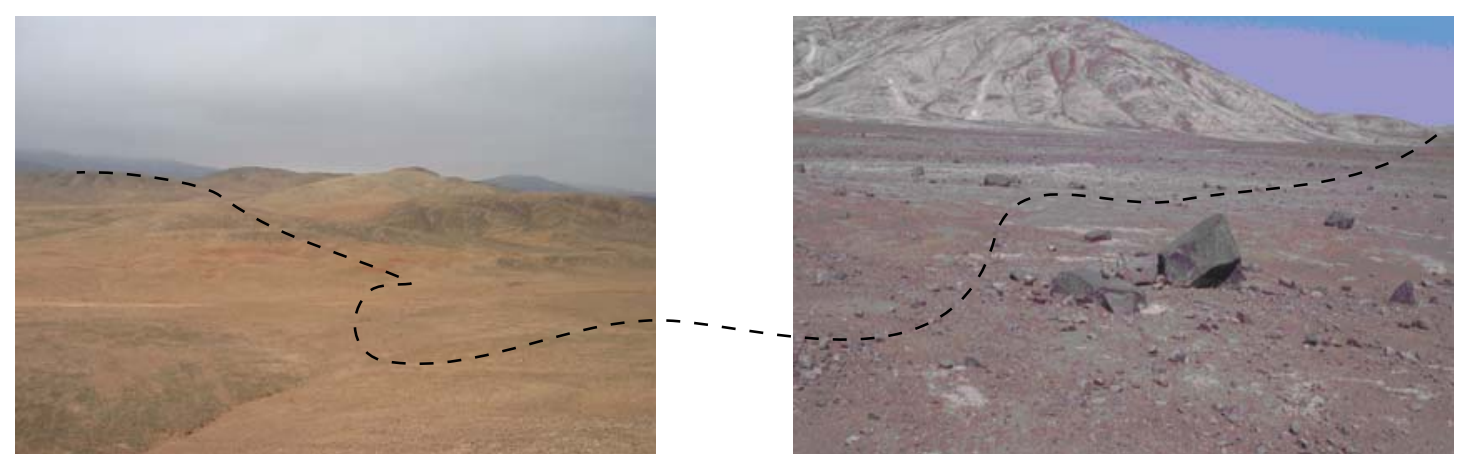

Figure 2. Biodiversity and distribution of habitats in Atacama subregions are not well understood. Where does life survive and where does it not? What factors govern the distribution? Scientific investigation of the distribution of life in the Atacama desert involves surveying organisms and identifying the variation in potential habitat, such as between coastal (left) and desert (right) environments. The concept of operation is to make robotic traverses deploying instruments that can make rapid assessment of geologic and biologic properties and survey accurately over long distances. 


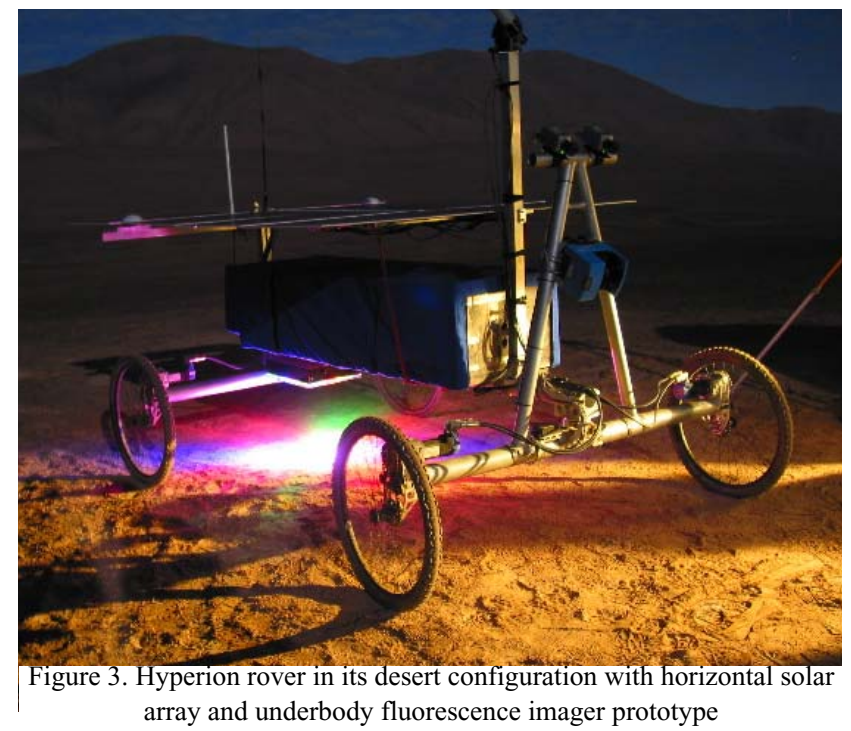

reduced complexity, and vertically-oriented solar panels.

For this research we transformed it for an equatorial desert environment, Fig. 3. Specifically we modified its solar array for horizontal orientation after simulation analysis of insolation (solar energy) indicated that without a known bias in direction of travel, a horizontal orientation would produce highest output and that a sun-tracking array was unnecessary and added complexity.

Hyperion was further modified with the addition of rollpitch sensors and a gyro to enable it to dead-reckon position (meaning without the use of GPS. Additional power monitoring sensors measure component-level power production and/or consumption.

Two devices were prototyped and incorporated to support a preliminary science investigation: a stereo panoramic imager and an underbody fluorescence imager.

\section{A. Mechanism}

The configuration of Hyperion is based on a premise that mechanical simplicity can reduce mass and thus reduce necessary system power. Our approach is to seek intelligent behavior to compensate constraints imposed by simplicity.

Hyperion, is capable of speeds up to $0.3 \mathrm{~m} / \mathrm{s}$. It is designed for natural terrain and to surmount obstacles up to $20 \mathrm{~cm}$ in height. It uses four wheel independent drive and passively articulated steering. This steering design combines the maneuverability of frame articulation with the mechanical simplicity of skid steering. The front axle angle is controlled by differential velocity on the front wheels, eliminating the need for a steering actuator. Velocity control on all four wheels eliminates skidding while minimizing locomotion power.[6]

TABLE 1: HYPERION SPECIFICATIONS

\begin{tabular}{|l|l|}
\hline Mass & $181 \mathrm{~kg}$ \\
\hline Size & $2.0 \mathrm{~m}$ width, $2.4 \mathrm{~m}$ length, 3.0 m height \\
\hline Speed & $0.25 \mathrm{~m} / \mathrm{s}$ nominal, $0.3 \mathrm{~m} / \mathrm{s}$ maximum \\
\hline Power & $120 \mathrm{~W}$ steady-state $+90-200 \mathrm{~W}$ locomotion \\
\hline
\end{tabular}

\section{B. Power}

The system to collect and store power for the robot consists of two arrays of solar cells, two maximum powerpoint trackers (MPPT), two sets of batteries, and components for conversion and distribution of power to the various subsystems, Fig. 4. Hyperion's solar arrays have 8 modules fabricated from (12.8\% efficient) Silicon cells. The total area of solar cells is $3.45 \mathrm{~m}^{2}$ which provides $400 \mathrm{~W}$ given isolation of $900 \mathrm{~W} / \mathrm{m}^{2}$, the typical daily maximum in the Atacama. Maximum power-point trackers control charge/discharge for varying insolation and thermal conditions and support two separate $24 \mathrm{~V}$ power buses. Two sets of lead acid gel-cell batteries maintain the power bus under conditions of shadowing or peak consumption during slope climbing. The batteries have capacity to run Hyperion for approximately 2 hours under typical operating conditions. The two battery buses also supply power to a main bus for the computing, sensing, control and communication subsystems. Power system parameters such as bus voltage, battery and load currents, are monitored.

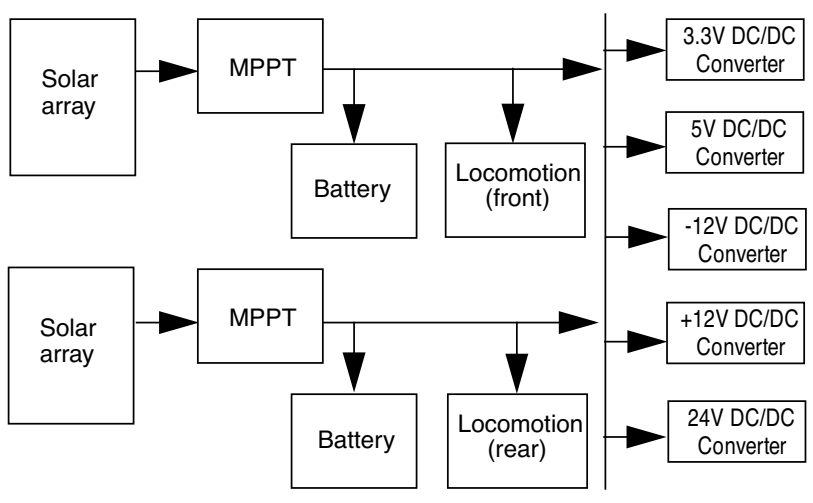

Figure 4. Hyperion power system

\section{Computing and Control}

The computing hardware is designed to support autonomous functionality such as stereo perception and local navigation. The computational complexity of robot autonomy can always make full use of high-performance processors yet to meet the competing demand for power efficiency, the computing system includes a single $500 \mathrm{MHz}$ Pentium III processor board in a $3 \mathrm{U}$ CompactPCI form factor. The on-board operating system is Linux.

The computing system interfaces over IEEE-1394 buses for devices including eight digital cameras and RS-232 buses for a motion controller, a laser rangefinder, a roll-pitch sensor, and the power management and distribution microcontroller. Hyperion's motion control system consists of two elements: control of drive motor velocities and control of steering axle angle; both are carried out by a multi-axis controller for PID drive motor control using encoder feedback. [6].

\section{Science Instruments}

Hyperion carries a Stereo Panoramic Imager, which is composed of two, color CCD cameras with $0.28 \mathrm{mrad} / \mathrm{pixel}$ resolution, $21^{\circ}$ field of view and $25 \mathrm{~cm}$ baseline, mounted atop a pan-tilt mechanism. This devices allows the remote science team to collect high-resolution stereo pairs of near- 
field objects as well as complete $360^{\circ}$ panoramas. This is used for remotely investigating geologic context and observing macroscopic organisms.

Fluorescence is the property of some molecules to emit light at longer wavelength than the wavelength that illuminates them; it is caused by the absorption and emission of energy by electrons composing the molecule. Chlorophyll, found in organisms that photosynthesize sunlight, fluoresces when illuminated by specific wavelengths of light. The Fluorescence Imager on Hyperion is a proof-of-concept prototype that uses blue and red highintensity LEDs and three simple CCD cameras each with a different band pass filters in front of the lens, Fig. 3.

\section{E. Autonomy Software}

Hyperion's software architecture exhibits a property of sliding autonomy so that the current conditions dictate the robot's behavior. A person can interact with the robot: by directly teleoperating its actions, by enabling it to safeguard their commands or by allowing it to navigate autonomously. For experiments in the Atacama we focused on autonomous operation and, as our objectives indicate, long-distance autonomous traverse with automated scientific sampling.

In its autonomous mode the Stereo Mapper classifies terrain, generating a traversability map from stereo imagery at $1.5 \mathrm{~Hz}$. The Navigator evaluates the map and selects a path that best leads the robot to the next goal. The State Estimator integrates odometry and inertial sensing to estimate rover position and orientation. The Health Monitor samples sensors and software variables to detect faults. The Mission Planner determines position and orientation goals and produces a scheduled plan, at $30 \mathrm{~m}$ resolution, to guide the Navigator along an energy and terrain feasible route and the simple Mission Executive parcels the plan out to the Navigator as the Health Monitor checks progress.

\section{E.1. Health Monitoring}

While exploring the unknown, circumstances will arise that challenge the capability of a robot. The robot must recover from errors as well as monitor developing conditions so that it may modify its behavior appropriately. This self-awareness becomes crucially important to

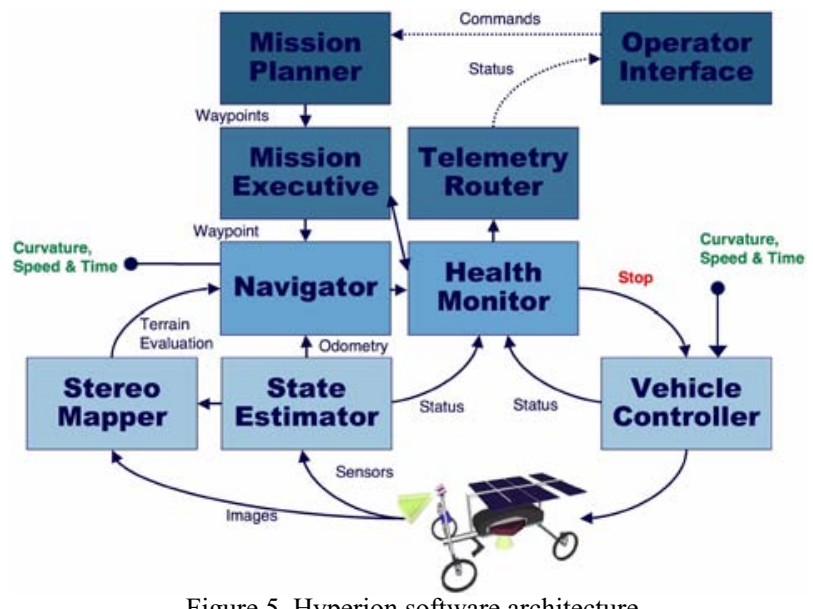

Figure 5. Hyperion software architecture achieving long-duration operation where faults should not end the mission.

In the Hyperion software system the Health Monitor samples approximately 100 state variables including temperatures, voltages, currents, positions, orientations, and velocities as well as monitors software state like process activity and uncertainties in stereo correlation and terrain modeling. Individually or in combination, the state variables can indicate the presence of a fault. The Health Monitor is being developed to recover the system when appropriate. On critical faults, the Health Monitor commands an emergency stop of the system and puts the rover in a safe mode until recovery instructions are received (safeguarded mode). When the rover is commanded into autonomous operation, the Health Monitor first checks for faults before allowing the robot to begin driving.

Hyperion's Health Monitor has been designed to address complex faults such as wheel entrapment or vehicle high centering, that require the inference from a number of observables. In the next phase we are creating a Rover Executive to generate appropriate actions in nominal and in fault conditions detected by the Health Monitor.

\section{E.2. Perception}

Critical to the embodiment of the Navigator are the characteristics of the terrain it is to operate on. Hyperion is designed to operate in terrain typical of the desert and of many regions of Mars and the Moon. The ground is gently sloping with discrete rocks and impassable gullies interspersed at a fairly low density. The surface generally consists of shattered rocks, hard-packed soil, and soft sands. This type of environment allows for many optimizations to the navigation algorithm that would be inappropriate in more dense terrain.

Hyperion uses stereo vision for terrain perception. The nature of the terrain encourages the use of an optimistic navigation algorithm. Terrain not seen by stereo vision is considered traversable for planning. In the past we have used a laser scanner to operate as a "virtual bumper", detecting obstacles missed by the optimistic evaluation and stopping the robot prior to collision.[3] However we have found that stereo vision alone is effective in sparselyfeatured natural environments, like the desert, if we treat the far-field optimistically (anticipating that unknown areas will be traversable) and the near-field pessimistically (requiring that the next motion be evaluated before moving forward). This combination allows for efficient navigation without undue risk or perfectly accurate terrain perception.

\section{E.3. Navigation}

The Navigator for Hyperion operates on a queue of waypoints. For each of these positions and orientations in the queue, a new map is initialized and commands are generated to drive the robot. Path evaluation is implemented using the $\mathrm{D}^{*}$ algorithm. [4] The basic procedure of the Navigator when evaluating possible paths to the next goal is:

- Update the robot position via the State Estimator

- Obtain stereo information from the Stereo Mapper 


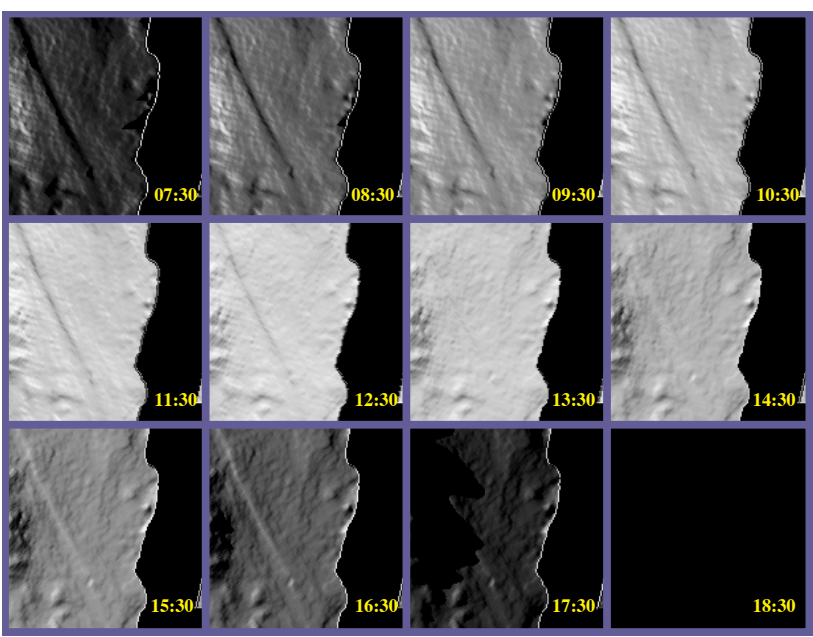

Figure 6. Solar lighting model developed from terrain elevation model and solar ephemeris and used to determine available energy.

- Insert the terrain information into the map

- Update position to account for movement

- Evaluate the cost along a discrete set of arcs

- Choose the arc that has the lowest value of total cost along the arc plus cost from the end of the arc to the goal

- Send radius, speed, and time for arc to the Controller

The robot travels a fraction of the chosen arc, and then the algorithm iterates, so smooth transition among discrete arcs is achieved.[5] Goals are regions whose dimensions and orientation were determined by scientists.

\section{E.4. Mission Planning}

The Mission Planner solves for time-sequenced path plans, with minimum energy guidelines, to enable the solarpowered robot to operate for extended periods. The robot must reason about the complex interactions between motion of the sun in the sky, terrain, shadows, solar array pointing and energy consumption.

The Mission Planner applies the TEMPEST algorithm [7] which combines goals with the ephemeris data with models of robot power performance and operational constraints to form a basis for path and time search. Surface lighting is determined by a ray-tracing algorithm that determines line of sight visibility from the sun to each cell on the map, Fig. 6

The planner uses an incremental search engine (ISE)[4] to determine the route that is optimal based on navigation and power criteria and outputs a list of waypoints and times that is transferred to the Navigator for mission execution.

\section{FIELD EXPERIMENT}

The experiments in the first field season focused on necessary in situ validation of individual components, instruments, algorithms, and models. Operational tests with the rover were conducted to investigate issues including perception, localization, and mobility. Tests will be conducted with scientific instruments to understand their methods of use and technical properties important to field deployment by a rover.

The function of the first field season is to pose and answer key questions that must be resolved to proceed to design of

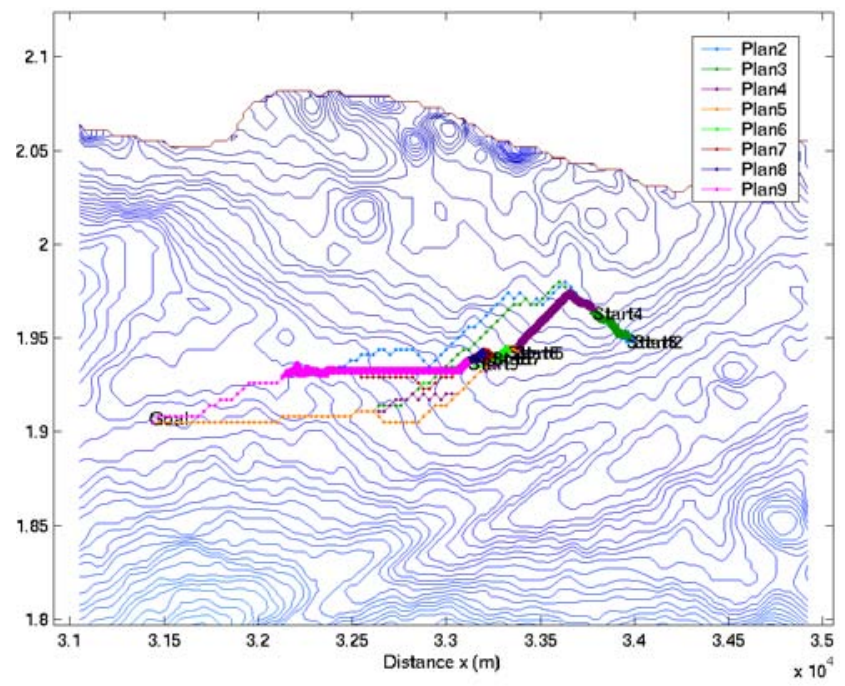

Figure 7. Mission plans and subsequent replans to reach a goal several kilometers from the start.

the robotic astrobiology system and to achieve three-year goal to with robotics detect and map the distribution of life in the Atacama Desert.

\section{A. Experiments}

The 2003 experiments were designed to: conduct a preliminary science investigation by collecting field samples of analysis and evaluating in situ several candidate instruments; measure rover performance including solar efficiency, mobility, perception, navigational reliability, localization accuracy, communication range, autonomy, and general environmental conditions; and lastly to specify requirements for a capable astrobiology rover.

\section{A.1. Localization}

We conducted experiments to determine localization accuracy in this environment using a variety of techniques. An accuracy of 3-5\% of distance traveled was achieved with a dead-reckoning algorithm that integrates wheel encoders, roll and pitch inclinometers and yaw gyro, Fig. 8 .

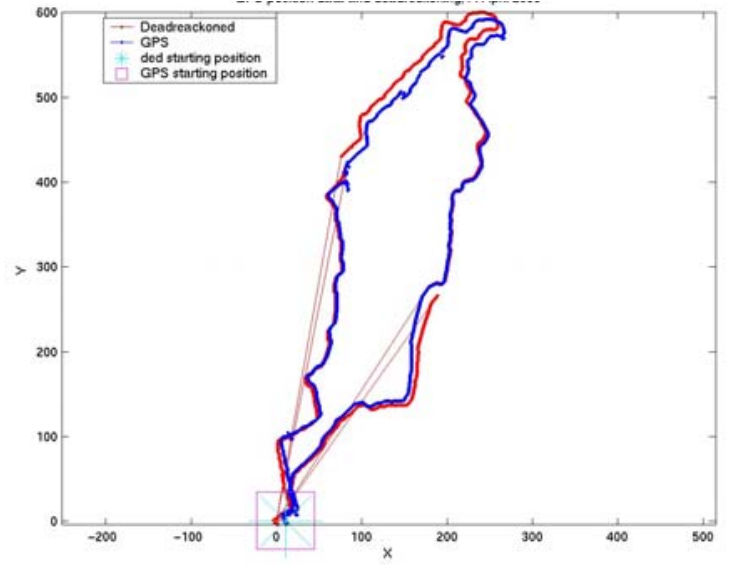

Figure 8. Dead-reckoned versus GPS ground-truth over three autonomous segments in desert (gently rolling, sparsely cluttered) terrain.

Experiments were conducted in determining orientation by sun tracking which proved accurate (typical error, $1^{\circ}$ ) and will be incorporated in the estimator to correct gyro drift. 


\section{A.2. Endurance Navigation}

Our purpose in conducting experiments in robotic traverse in the relevant environment was to validate the navigation approach and identify research priorities. We conducted autonomous traverses using only orbital data and planetary relevant communication and power. When the rover was unable to recover a from a fault or when it reached its goal, the experiment was concluded and statistics recorded. In total, $18 \mathrm{~km}$ of autonomous traverse was executed in 90 experiments. Each traverse was initiated by a single command to the Mission Planner which determined the path and guided the Navigator. The average distance per traverse was $200 \mathrm{~m}$ and the average speed was $0.25 \mathrm{~m} / \mathrm{s}$. There were 8 traverses that exceeded $300 \mathrm{~m}$ and one traverse that exceeded $1 \mathrm{~km}(1118 \mathrm{~m})$ with a single command. This milestone distance is one of the long-term objectives of this project, to enable planetary rovers to autonomously travel over $1 \mathrm{~km}$ per command cycle.

\section{A.3. Autonomy}

Rover autonomy was tested in 90 autonomous traverse experiments. The average traverse distance was $200 \mathrm{~m}$ and the termination condition was tied to a number of recoverable faults, Fig. 9. Common traverse-ending faults: include roll/pitch limit, no path ahead, and off schedule.

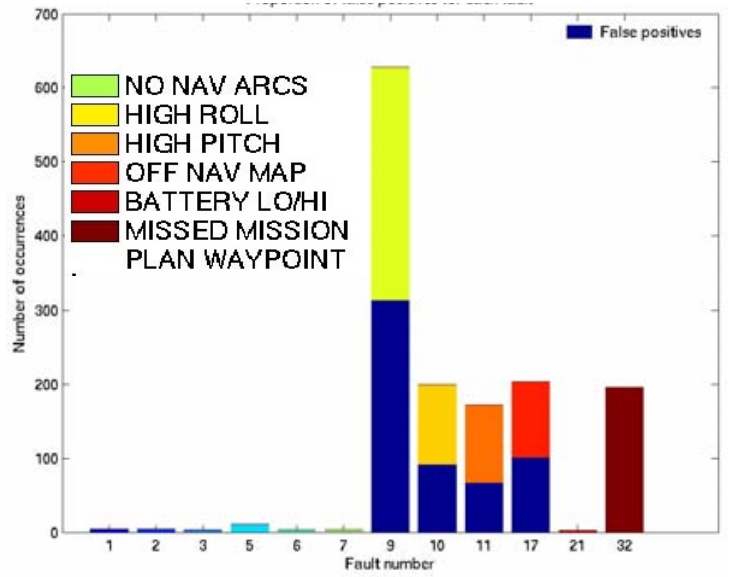

Figure 9. Faults detected in 90 autonomous traverse experiments

\section{CONCLUSION}

From an astrobiological standpoint the major conclusion of the 2003 Atacama field experiment is that all tests indicate that it is feasible to deploy the necessary biologic and geologic instruments with a rover and to successfully detect life and characterize habitat. The detection of life was not accomplished unambiguously in the first field season but indications are that with appropriate instruments and rover it will be possible.

From a robotics view, the major result is the accomplishment of single-command, one-kilometer traverse through planetary-analog terrain. In the coming field seasons, we intend to further develop and rigorously test our approach, but at least one instance has been demonstrated.

\section{A. Rover Requirements}

To support the intended science investigation, we have identified the following critical issues and modifications required for the system design:

- Accommodate science instrument payload

- Incorporate translation motions for imager

- Increase solar array power output

- Increase battery capacity for night operations

- Increase computation for more complex planning

- Add low power and switched electronics

- Increase rover speed to decrease traverse time

- Increase wheel torque to improve slope climbing

- Eliminate drivetrain hysteresis to improve control

- Incorporate subsurface access mechanism

- Autonomously recover from faults.

\section{B. Continuing Research}

The Life in the Atacama project will continue through 2006 with field experiments planned in each year. In the next field season a refined rover design with greater mobility and speed and integrated science instruments will be deployed.

Our robotics research efforts will focus on energyefficient, terrain-capable rover designs, far-field terrain evaluation to evaluate farther ahead (5-30 m), fault detection and recovery with self diagnosis and no interruption of plans, mission plan with continuous replanning, and resource-cognizant mission execution.

\section{ACKNOWLEDGMENTS}

This paper describes the work of the Life in the Atacama project to which all members have made important contributions. We acknowledge and thank Dimi Apostolopoulos, Francisco Calderón, Guillermo Chong, Peter Coppin, Matt Deans, Allan Lüders, Dana Martinelli, Reid Simmons, Sanjiv Singh, Trey Smith, Tony Stentz, Dennis Strelow, and Alan Waggoner. We thank the NASA Astrobiology Science and Technology for Exploring the Planets (ASTEP) program, David Lavery, Program Executive and Michael Meyers, Program Scientist.

\section{REFERENCES}

[1] Navarro-Gonzalez R, et. al., Mars-like soils in the Atacama Desert, Chile, and the dry limit of microbial life, Science 302(5647): 101821, November 2003.

[2] Banin, A. Clark, B., W nke, H. Surface Chemistry and Mineralogy, Mars, Univ. of Arizona Press, Tucson, 1992.

[3] D. Wettergreen, M. Dias, B. Shamah, J. Teza, P. Tompkins, C. Urmson, M. Wagner, W. Whittaker, First Experiment in Sun-Synchronous Navigation, ICRA, Washington D.C., May 2002.

[4] A. Stentz, "The Focused D* Algorithm for Real-Time Planning", IJCAI, Montreal, Canada, August 1995.

[5] C. Urmson, M. Dias, R. Simmons, "Stereo Vision Based Navigation for Sun-Synchronous Exploration," IROS 2002, September, 2002.

[6] B. Shamah, et. al., Steering and Control of a Passively Articulated Robot, SPIE Robotic Systems, Boston, October 2001.

[7] P. Tompkins, A. Stentz, W. Whittaker, Mission Planning for the SunSynchronous Navigation Field Experiment, ICRA 2002, Washington D.C., May 2002.

[8] D. Wettergreen, et. al., Life in the Atacama Investigation 2003: Experimental Plans and Technical Results Robotics Institute Technical Report CMU-RI-TR-03-50, December 2003. 